\title{
Role of intestinal microbiota in cardiovascular disease risk in end stage renal disease patients
}

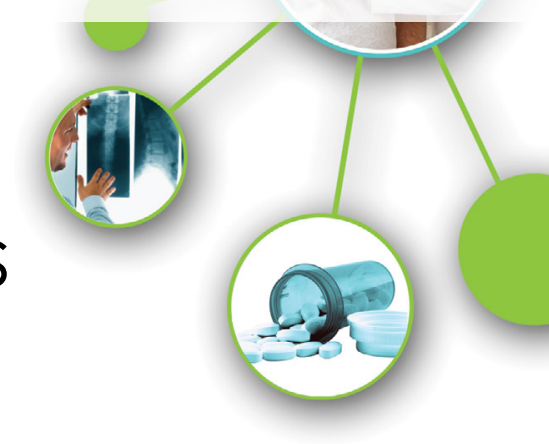

Chronic inflammation is considered as a non-traditional risk factor for cardiovascular mortality in the chronic kidney disease (CKD) population. Recent studies have revealed that alterations in gut microbiota composition and intestinal barrier have been associated with inflammation and oxidative stress in CKD patients which in turn promotes adverse cardiovascular outcomes and CKD progression. The purpose of our study was to assess the alteration of beneficial gut microbiota mainly Lactobacillus acidophilus in fecal samples of patients with end stage renal disease (ESRD) and the extent of its effect by the co-existence of cardiovascular complications. Forty patients with ESRD, another forty ESRD with CVD and 20 healthy adults participated in the study. The fecal composition of Lactobacillus acidophilus was identified using de Man Rogosa Sharp agar followed by further confirmation using polymerase chain reaction technique. A significant abundance of Lactobacillus acidophilus in ESRD patients compared to controls was noticed, and this increase was detected mainly in ESRD patients with CVD. Furthermore, Lactobacillus acidophilus was not correlated significantly with serum cholesterol, triglycerides or urinary protein creatinine ratio. In conclusion, Lactobacillus acidophilus as a beneficial microbiome has no protective role against progression of CKD and CVD risks. Thus, the exact role of gut microbiota in CKD progression has not been yet fully elucidated and further investigation will be needed.

\section{Keywords: gut microbiota, Lactobacillus acidophilus, ESRD, CKD, CVD}

\section{Introduction}

Chronic kidney disease (CKD) patients have many risk factors for cardiovascular disease (CVD), with a high rate of cardiovascular mortality [1]. These excess rates of CVD are related to both atheromatous and nonatheromatous vascular affection, whether it is traditional or non-traditional risk factors which are responsible of this vascular injury. Among the possible treatable traditional risk factors are hypertension, diabetes, anemia, dyslipidaemia, smoking and obesity [2]. However, CKD patients have a high prevalence of what is called 'emerging risk factors' e.g. hyperhomocysteinemia, hypovitaminosis D, oxidative stress and inflammation [3].

The existence of large community of microbes in the intestinal tract constitutes a dynamic and symbiotic ecosystem that is in continuous interaction with the host metabolism [4-6]. Among the important role of the gut microbiota in healthy individuals is regulation of different aspects of immune system, protection of the host against pathogenic microbes and production of vitamins and other essential nutrients [7].

Firmicutes, Bacteroidetes, Actinobacteria, Proteobacteria and Verrucomicrobia represent the main five bacterial phyla that resident in the human gut and the most dominant of these are, Bacteroidetes (Bacteroides, Prevotella and Xylanibacter) and Firmicutes (Ruminococcus, Clostridium, Lactobacillus, Eubacterium, Faecalibacterium and Roseburia) [8].

The Firmicutes/Bacteroidetes ratio is considered as an important factor in the composition of the intestinal microbiota $[9,10]$ and any change in this ratio increases susceptibility to infections, immune disorders, inflammation, oxidative stress and insulin resistance.

Vaziri et al demonstrated that CKD and its therapeutic interventions, including prevention of hyperkalemia via dietary restriction of fruits, vegetables, and high-fiber products, as well as, oxalate overload, use of phosphate binding agents to manage hyperphosphatemia, and administration of antibiotics to treat vascular
Ashraf Okba ${ }^{1}$, Rasha Shahin ${ }^{1}$, Aber Baki ${ }^{1}$, Dina Sheha ${ }^{1}$, Mohamed Mekawy² \& Motaz Farag 3 ${ }^{1}$ Department of Internal Medicine, Allergy and Clinical Immunology, Faculty of Medicine, Ain Shams University, Cairo, Egypt

${ }^{2}$ Department of Clinical Pathology, Faculty of Medicine, Ain Shams University, Cairo, Egypt

${ }^{3}$ Internal Medicine Unit, Police Hospitals, Cairo, Egypt

*Author for correspondence: rashayms@med.asu.edu.eg 
access, can significantly alter the biochemical milieu of the intestinal tract and thus may alter the structure, composition, and function of microbial flora [11].

The loss of kidney function in CKD leads to urea secretion into the gastrointestinal tract. Subsequent hydrolysis of urea by urease expressed by some gut microbes, leads to the formation of large quantities of ammonia that could affect the growth of commensal bacteria [12]. Subsequently, these events can contribute to uremic toxicity, inflammation, and cardiovascular, nutritional, and other complications of CKD. Other contributing factors that could alter the growth of intestinal microbiota include slow colonic transit [13], metabolic acidosis [14], intestinal wall edema [15] and possibly oral iron intake [16].

Lactic acid bacteria (LAB) such as Lactobacillus acidophilus are considered to be beneficial microorganisms and have been associated with multiple potential health effects in both humans and animals [17]. Among these effects, the hypocholesteremic effect of Lactobacillus acidophilus has recently gained interest due to its potential role in the prevention of atherosclerosis.

Moreover, the use of Lactobacillus acidophilus strain LB as probiotic was associated with reduced bacterial translocation in the uremic rats and increased macrophage phagocytosis. This strain is not only considered as antibacterial [18], but also reduces epithelial barrier loss induced by pathogenic bacteria such as the Escherichia coli strain C1845 [19].

For this purpose, the present study was designed to assess the alteration of beneficial gut microbiota mainly Lactobacillus acidophilus in fecal samples of Egyptian patients with ESRD and the extent of its effect by co-existence of cardiovascular complications in comparison to healthy controls.

\section{Material and methods}

This case control study included 100 adult subjects; being categorized into 3 groups; first group of 20 healthy subjects as controls, second group of $40 \mathrm{ESRD}$ patients with no cardiovascular diseases, and third group of 40 ESRD patients with established cardiovascular diseases were recruited in the period from May 2014 to May 2016 from the hemodialysis unit of the Ain Shams University hospitals, Cairo, Egypt. The healthy subjects were not on any medications and had no established medical problems. The Human Research Ethics Committee of Ain Shams University hospital approved this study and all participants gave informed consent.

According to KDIGO CKD classification 2012, ESRD subjects were eligible for the study inclusion if the estimated glomerular filtration rate $(\mathrm{eGFR})<15 \mathrm{~mL} / \mathrm{min} / 1.72 \mathrm{~m}^{2}$, and they were on hemodialysis [20,21].

CVD was diagnosed if the participant had one or more of the following: echocardiographic abnormalities (systolic dysfunction, diastolic dysfunction or left ventricular hypertrophy) and past history of myocardial infarction, or heart failure. Exclusion criteria included: a) known chronic gut-related diseases, (b) patients who underwent bariatric surgeries, (c) patients with end-stage liver disease, and (f) patients who were treated with antibiotics, prebiotics or probiotics for at least three consecutive days for any reason during one month prior to the study enrollment.

The subjects' demographics, body mass index (BMI), history of hypertension, mean arterial blood pressure (MABP), and drug history of either antihypertensive medication or statins were recorded. In addition all patients were investigated before their hemodialysis session for the following: hemoglobin levels $(\mathrm{Hb})$, cholesterol, triglycerides, low density lipoprotein cholesterol (LDL-C), high density lipoprotein cholesterol (HDL-C), uric acid, ferritin, urinary protein creatinine ratio (UPCR), and eGFR. Also, echocardiography was done for the patients.

\section{Stool culture for Lactobacillus acidophilus}

Stool culture on de Man Rogosa Sharp agar (MRS), after sample collection from the patients. Quantitative culture for Lactobacillus acidophilus (in colony forming unit per gram [CFU/g] of stools) was done by weighing 1 gram of the stool specimen diluted in $2 \mathrm{ml}$ of sterile saline.

Serial dilutions of this suspension were prepared in sterile saline solution; $1 \mathrm{ml}$ of each dilution was inoculated into MRS agar plates 
using a standard loop. Incubation of the plate was done anaerobically at $37^{\circ} \mathrm{C}$ for $2-3$ days. Colony forming unit per gram was detected for each stool specimen using the following formula:

$$
\mathrm{CFU} / \mathrm{Gm}=\mathrm{N} \times \mathrm{D} \times 2
$$

\section{W}

Where CFU: colony forming unit, Gm: gram, $\mathrm{N}$ : the number of colonies on the plate, D: dilution, W: the weight of the stool specimen in $\mathrm{Gm}$, and 2: the original dilution of the stool specimen.

\section{DNA extraction and sequencing for Lactobacillus acidophilus from stools}

Stool samples were collected from ESRD patients and healthy subjects. All samples were processed for DNA extraction within $24 \mathrm{~h}$ after receipt.

\section{DNA isolation}

DNA from Lactobacillus acidophilus in the stool samples was extracted using proteinase $\mathrm{K}$ protocol after centrifugation of the broth for 2 min. Briefly, bacterial cells were re-suspended in 467 (1 of freshly prepared $(50 \mathrm{mM}$ Tris$\mathrm{HCl}, 10 \mathrm{Mm}$ EDTA, PH 7.5) $30 \mathrm{UL}$ of $10 \%$ SDS and $3(1$ of $20 \mathrm{mg} / \mathrm{ml}$ proteinase-k, Sigma Aldrich. Following incubation at $65^{\circ} \mathrm{C}$ for 45 min with shaking, the DNA was obtained by phenol/chloroform/isoamyl alcohol (25:24:1) precipitated with isopropanol washed with ethanol (70\%) and dried under vacuum [22]. DNA pellet was resuspended in 25 /1 of sterile distilled water and stored at $-20^{\circ} \mathrm{C}$.

\section{- PCR primer}

DNA pellet was confirmed to be of bacterial origin using the universal $16-S$ primer. F (5AGA GTT TCC TGG CTC TSA AG-3) and R (5-ACG ACC TTG TTA GMT CGA CTT3) that amplify a 1500 bp fragment. The genous specific primers of identification of Lactobacilli were designed on 16SrRNA as described by Dubernet et al [23].

The genus specific primers, (Sigma, USA) for Lactobacilli amplifies a $123 \mathrm{bp}$ fragment. LbL MA1-rev (5-CTC AAA ACT AAA CAA AGT TTC-3) and R16-1 (50-CTT GTA CAC ACC GCC CGT CA-3).

\section{- PCR conditions and procedure}

PCR reaction mixture $(25$ (l) was composed of 25 pmol of each primer, $0.2 \mathrm{mM}$ of each dNTP, $1 \times$ PCR buffer $50 \mathrm{mM}$ Tris- $\mathrm{HCl}, \mathrm{PH}$ 8.8, $2.5 \mathrm{mM} \mathrm{MgCl}_{2}, 15 \mathrm{Mm}\left(\mathrm{NH}_{4}\right)_{2} \mathrm{SO}_{4} 0.45 \%$ Triton X-100, $10 \mathrm{ng}$ of bacterial DNA and $1 \mathrm{U}$ of Taq DNA Polymerase (Biotech International, Australia).

The PCR was carried out in a ROTOR Gene $\mathrm{Q}$ (Berlin, Germany) following the manufacture's instruction. The amplification program is as follows: initial denaturation at $95^{\circ} \mathrm{C}$ for $5 \mathrm{~min}$, followed by 30 cycles consisting of denaturation at $95^{\circ} \mathrm{C}$ for $30 \mathrm{~s}$, annealing at $55^{\circ} \mathrm{C}$ for $30 \mathrm{~s}$, extension at $72^{\circ} \mathrm{C}$ for $30 \mathrm{~s}$, and a $7 \mathrm{~min}$ final extension step at $72^{\circ} \mathrm{C}$. Amplification products were subjected to electrophoresis in $1 \%$ agarose gels (Electrophoresis grade, Invitrogen) in TAE buffer (40 mM Tris acetate, $1 \mathrm{mM}$ EDTA, $\mathrm{pH}$ 8.2) followed by ethidium bromide staining $(5 \mathrm{~g} / \mathrm{ml}$ ) and visualized under UV light. The strain Lactobacillus Acidophilus ATCC 4356 was used as positive control.

\section{Statistical analysis}

Data were analyzed using SPSS (Statistical Program for Social Sciences) version 19. Biomarker values were expressed as mean \pm standard deviation (SD), median, and interquartile ranges. Comparison of two groups was analyzed using Student's t-test and Wilcoxon Rank Sum test for parametric and non-parametric data respectively. Oneway analysis of variance (ANOVA) with post Hoc analysis (Tukey's test) was used for the comparison of three groups. Chi-square test was used to compare categorical data. Ranked Spearman correlation was used for measuring the correlation between the different variables. A p-value of less than 0.05 was considered significant.

\section{Results}

Demographic and clinical characteristics of the studied groups are shown in TABLE 1.

The review of antihypertensive medication in both ESRD groups showed that almost $70-75 \%$ were on either mono therapy or combined treatment of antihypertensive drugs. Approximately $70 \%$ used statins in their treatment regimen.

ESRD patients with CVD exhibited significant elevation of HDL-C, triglycerides while UPCR was significantly increased in those 
Table 1. Demographic and clinical characteristic of the studied groups.

\begin{tabular}{|l|c|c|c|}
\hline Parameters & Control group N=20 & \multicolumn{1}{c|}{ ESRD without CVD N=40 } & ESRD with CVD N=40 \\
\hline Age (years) & $45.4 \pm 3.5154$ & $47.2 \pm 11.3051$ & $44.225 \pm 10.4648$ \\
\hline Gender, $\mathrm{n}(\%)$ & $14(70 \%)$ & $26(65 \%)$ & $31(77.5 \%)$ \\
\hline Male & $6(30 \%)$ & $14(35 \%)$ & $9(22.5 \%)$ \\
\hline Female & $28.36 \pm 1.3508$ & $29.32 \pm 1.8747$ & $29.673 \pm 1.838$ \\
\hline $\mathrm{BMI}\left(\mathrm{kg} / \mathrm{m}^{2}\right)^{*}$ & $78.875 \pm 11.3503$ & $80.8 \pm 10.2887$ & $86 \pm 9.4032$ \\
\hline MABP $^{* *}(\mathrm{mmHg})$ & &
\end{tabular}

All values are expressed as mean \pm SD except gender which is expressed as no. (\%).

$\mathrm{BMI}$, body mass index; MABP, mean arterial blood pressure

*BMl: controls vs. ESRD without CVD, $p<0.001$; controls vs. ESRD with CVD, $p<0.001$; ESRD without CVD vs. ESRD with CVD, $p=0.952$

${ }^{* *} \mathrm{MABP}$ : controls vs. ESRD without CVD, $\mathrm{p}<0.001$; controls vs. ESRD with CVD, $\mathrm{p}<0.001$; ESRD without CVD vs. ESRD with CVD, $p<0.001$

\section{Table 2. Laboratory parameters of the ESRD studied groups.}

\begin{tabular}{|c|c|c|c|}
\hline Parameters & ESRD without CVD $N=40$ & ESRD with CVD N=40 & P-value \\
\hline $\mathrm{Hb}(\mathrm{gm} / \mathrm{dl})$ & $9.805 \pm 1.5147$ & $9.785 \pm 1.4181$ & 0.744 \\
\hline Triglycerides(mg/dl) & $155.5(79.5-193)$ & $211(176.5-231)$ & $<0.001$ \\
\hline T.Cholesterol(mg/dl) & $185.5(143-226)$ & $211(178-231)$ & 0.02 \\
\hline $\mathrm{HDL}-\mathrm{C}(\mathrm{mg} / \mathrm{dl})$ & $54(43-65)$ & $71(54-100)$ & 0.001 \\
\hline $\mathrm{LDL}-\mathrm{C}(\mathrm{mg} / \mathrm{dl})$ & $108.5(64.25-122.75)$ & $111.5(76-132)$ & 0.114 \\
\hline Ferritin (ng/ml) & 239.5(126.75- 557.75) & $243.5(205-537.25)$ & 0.61 \\
\hline Serum uric acid $(\mathrm{mmol} / \mathrm{L})$ & $5.85(5-6)$ & $5(4-6)$ & 0.029 \\
\hline $\mathrm{UPCR}(\mathrm{mg} / \mathrm{mg})$ & $4388(3326.25,5619)$ & $561(449.25,2261)$ & $<0.001$ \\
\hline eGFR(ml/min) & $6(5,8)$ & $5(5,6)$ & 0.952 \\
\hline
\end{tabular}

All values are expressed as median, interquartile except for $\mathrm{Hb}$ which is expressed as mean $\pm \mathrm{SD}$

$\mathrm{Hb}$, hemoglobin; $\mathrm{HDL}$, high density lipoprotein; LDL, low density lipoprotein; UPCR, urinary protein creatinine ratio; eGFR, estimated glomerular filtration rate.

\begin{tabular}{|c|c|c|c|c|}
\hline Parameter & $\begin{array}{c}\begin{array}{c}\text { Control group } \\
\mathrm{N}=\mathbf{2 0}\end{array}\end{array}$ & $\begin{array}{l}\text { ESRD without CVD } \\
\qquad N=40\end{array}$ & $\begin{array}{c}\text { ESRD with CVD } \\
\qquad N=40\end{array}$ & P-value \\
\hline Lactobacillus acidophilus count (CFU/gm) & $24.66 \pm 3.13$ & $27.57 \pm 3.77$ & $30.47 \pm 2.72$ & $<0.001$ \\
\hline \multicolumn{5}{|l|}{ Tukey's test } \\
\hline $\begin{array}{l}\text { Healthy Control vs. } \\
\text { ESRD without CVD } \\
P<0.001^{* *}\end{array}$ & \multicolumn{2}{|c|}{$\begin{array}{l}\text { Healthy Control vs. } \\
\text { ESRD with CVD } \\
P<0.001^{* *}\end{array}$} & \multicolumn{2}{|c|}{$\begin{array}{l}\text { CKD without vs. } \\
\text { ESRD without CVD } \\
P<0.001^{* *}\end{array}$} \\
\hline
\end{tabular}

Table 4. Correlation of Lactobacillus acidophilus count and the various studied parameters among ESRD without and with CVD complications

\begin{tabular}{|c|c|c|c|c|}
\hline \multirow{2}{*}{ Parameters } & \multicolumn{2}{|c|}{ ESRD without CVD } & \multicolumn{2}{|c|}{ ESRD with CVD } \\
\hline & $r$ & P-value & $r$ & P-value \\
\hline Age (years) & -0.005 & 0.974 & 0.046 & 0.776 \\
\hline BMI (kg/m2) & -0.252 & 0.117 & -0.193 & 0.234 \\
\hline $\mathrm{MABP}(\mathrm{mmHg})$ & -0.228 & 0.704 & -0.123 & 0.448 \\
\hline Triglycerides (mg/dl) & 0.313 & 0.049 & -0.159 & 0.328 \\
\hline T.Cholestrol (mg/dl) & -0.177 & 0.275 & -0.021 & 0.897 \\
\hline HDL-C(mg/dl) & 0.016 & 0.924 & 0.05 & 0.76 \\
\hline LDL-C (mg/dl) & -0.252 & 0.117 & -0.155 & 0.338 \\
\hline eGFR (ml/min) & -0.168 & 0.301 & -0.114 & 0.482 \\
\hline U-PCR (mg/m) & -0.025 & 0.88 & -0.153 & 0.347 \\
\hline
\end{tabular}

BMI, body mass index; MABP, mean arterial blood pressure; HDL-C, high density lipoprotein cholesterol; LDL-C, low density lipoprotein cholesterol; UPCR, urinary protein creatinine ratio; eGFR, estimated glomerular filtration rate.

patients ESRD without CVD complications as shown in TABLE 2.
The difference in the incidence of detected Lactobacillus acidophilus in stool samples of 
ESRD versus healthy subjects was statistically significant at $\mathrm{p}<0.001$. Further analysis revealed that Lactobacillus acidophilus count was higher among ESRD patients with CVD complications than ESRD without CVD complications (TABLE 3).

On assessing the correlation between Lactobacillus acidophilus and various studied parameters among ESRD patients, Lactobacillus acidophilus count did not differ significantly with age, BMI, MABP as well as various metabolic parameters (TABLE 4), but a weak non-significant negative correlation was observed with triglycerides, total cholesterol, LDL-C as well as eGFR and U-PCR.

\section{Discussion}

It has been strongly suggested that the gut microbiome is an important factor in the maintenance of health [24], and intestinal dysbiosis that accompanies CKD allowed the development of oxidative stress and inflammation, which is a contributing factor, among other factors, to CVD risk in CKD patients [25].

To best of our knowledge, this study represent the first one in Egypt that assess the alteration of Lactobacillus acidophilus as a representative of beneficial gut microbiota in fecal samples of ESRD patients on hemodialysis and study the extent of its effect especially on those with CVD. Surprisingly, we found that there is abundance of Lactobacillus acidophilus in ESRD patients compared to healthy control. Moreover, there was relative abundance of Lactobacillus acidophilus in ESRD patients with CVD rather than ESRD patients without CVD. Our findings may be accounted for by the fact that all ESRD patients included in the study were on regular hemodialysis, which may have a positive effect on restoring gut symbiosis, thus introducing a novel therapeutic strategy that may ameliorate the harmful effect of dysbiosis. In addition, we supposed that, differences in the underlying systemic diseases, medications and, particularly, dietary restrictions as opposed to uremia could have modified the structure and function of the gut microbiome in those patients with ESRD. Furthermore, little is known about the genetic basis of the microbiome and how many genetic differences exist between enterotypes of gut microbiota within or between populations. A recent Chinese study concluded that considerable differentiation was observed between enterotypes and among healthy Chinese populations that was reflected in both the taxonomic composition as well as the genetic makeup of their functional genes, which could have been influenced by a variety of factors, such as diet and host genetic variation [26].

Alteration of colon microbiota is observed under uremic conditions. In contrast to our findings, early in 1996, Hida et al., using traditional plating methods for the analysis of fecal samples, did not detect any significant differences in the total number of colonic bacteria in hemodialysis patients compared to healthy individuals [27]. Moreover, it has been recently demonstrated that there is no change in level of Lactobacillus acidophilus in the stomach of CKD patients [25].

Furthermore, Wang et al. were the first to analyse the gut microbiota by real-time PCR and found that different Bifidobacterium species as B. catenulatum, B. longum, B. bifidum, Lactobacillus plantarum and Lactobacillus paracase $i$ were found at lower rates in peritoneal dialysis patients versus healthy individuals [28].

Vaziri et al. demonstrated via microarray analysis that there was a significant difference in the abundance of 190 microbial operational taxonomic units (OTUs) between the ESRD and the normal control individuals, who presented an increased abundance of Enterobacteriaceae, particularly certain clusters of Escherichia coli sequences [11].

Another study by Wong et al supported the hypothesis that CKD patients may have an abundance of bacterial families possessing urease, uricase, and indole- and p-cresol forming enzymes such as Clostridiaceae, Enterobacteriacea and Verrucomicrobiacea, which lead to the uraemic toxicity and systemic inflammation in these patients and a reduction of bacterial families possessing butyrateforming enzymes, including Lactobacillus and Prevotellae [29].

Another data from a Brazilian study confirmed an alteration of the gut microbiota composition in patients with stage III-IV CKD, where a reduction of fecal microbial diversity was observed upon the PCR-denaturing gradient gel electrophoresis of $16 \mathrm{~S}$ rRNA gene fragments in CKD patients when compared with healthy 
individuals [30]. Similarly, an experimental study in Japan analyzing the species of gut microbiota and found that the Lactobacillus population was reduced under CKD conditions [31].

Therefore, recent findings demonstrated that accumulation of metabolites of gut microbiota secondary to imbalance of gut microbiota together with changes in colonic epithelium may contribute to the progression of CKD and associated cardiovascular disease [32,33]. Toxic gases, indoxyl sulphate, p-cresyl sulphate, amines, ammonia and trimethylamine n-oxide (TMAO) as well as precursors for lipopolysaccharides (LPS) may be absorbed into the bloodstream and be responsible for activation of innate immunity, promotes the inflammatory state associated with CKD and, increases the incidence of cardiovascular disease $[8,30]$. Indeed, several studies have shown that these toxins could be reliable markers of cardiovascular disease and mortality in CKD patients [34,35]. Moreover, Celik et al. [36] who focused on the alterations of the small intestine in patients with chronic heart failure $(\mathrm{CHF})$, stated that increased bowel wall permeability and reduced intestinal resorption function secondary to the bowel wall edema and disturbed microcirculation in the gut are thought to be responsible for malabsorption of nutrients in patients with chronic heart failure and contribute to malnutrition and cardiac cachexia as well as it may lead to the translocation of bacteria and/or endotoxin, which may be an important stimulus for inflammatory cytokine activation in CHF. In addition, Sandek et al. detected a lack of immunological defense with an increased in bacterial biofilm and inflammatory response in CHF [37].

In recent years, there is a growing interest in restoring the symbiosis of intestinal microflora in $\mathrm{CKD}$ aiming to reduce the generation of uraemic toxins, oxidative stress, and inflammation. The investigation on efficacy of probiotics to decrease production of uremic toxin and to improve renal function has been performed in some human CKD studies [38]. Ranganathan et al. [39] observed an improvement in quality of life and a reduction in serum uric acid and creatinine levels in non-dialysed CKD patients after a probiotic supplementation. In contrast, Hyun et al. [40] showed that there was no significant effect of probiotics on the reduction of uraemic toxins in paediatric dialysis patients. Wang et al. [41] reported that after 6 months of probiotic supplementation in peritoneal dialysis patients, a reduction on serum levels of pro-inflammatory cytokines was observed. Studies with prebiotics are limited. Meijers et al. [42] observed significant reduction of p-cresyl sulphate levels in haemodialysis patients after a 4-week prebiotic oligofructose-inulin supplementation. Recently, Vaziri et al. [43] showed that prebiotic supplementation as a high resistant starch diet retards CKD progression and attenuates oxidative stress and inflammation in $\mathrm{CKD}$ rats.

Concerning blood lipid in the present, we couldn't find any significant correlation between Lactobacillus acidophilus composition and blood lipid in ESRD patients. However, unexpectedly, we observed increase levels of HDL-cholesterol in ESRD patients with CVD compared to ESRD patients without CVD. This finding could be explained by a Japanese study that excess oxidative stress may have gave rise of dysfunctional HDL in patients with ESRD and these patients with high HDL- cholesterol under these conditions may have enriched oxidized HDL which can result in increased CVD morbidity and mortality [44].

This study has several limitations, namely a relatively small study group, not considering other gut microbiota composition with limitation to lactobacillus acidophilus only. In addition to that, strict dietary restrictions that our ESRD patients were followed must have contributed to the observed changes in their microbial flora. Patients with CKD are polymedicated, other drugs rather than antibiotics, prebiotic and probiotic should be considered as proton pump inhibitor, antidepressant, statins may be associated with distinct gut microbiota changes [45]. Other medication that may also slow intestinal transit, phosphorus binders, ion exchange resins [13], or iron supplements that may have an effect on microflora but it is not well defined $[46,47]$.

In summary, it is known that the imbalance in the composition of the intestinal microbiota in $\mathrm{CKD} / \mathrm{ESRD}$ patients may represent another non-traditional risk factor because this imbalance can contribute to an increase in chronic inflammation and oxidative stress and CVD complications. Unfortunately, our 
present findings cannot support the findings of considering Lactobacillus acidophilus as having a reno-protective effect in ESRD patients. Further studies performed on a larger study population in Egypt, and including a wide diversity of microbiomes, are necessary to confirm this association. Also the requirements of studies that correlate the existence of Lactobacillus acidophilus to various inflammatory markers in CKD are mandatory. Accordingly, interventions aiming to restore and maintain the balance and function of the intestinal microbiota are extremely important. 
REFERENCES

Siasos G, Tousoulis D, Michalea S, et al. Biomarkers determining cardiovascular risk in patients with kidney disease. Curr. Med. Chem. 19(16), 2555-2571 (2012).

Kono K, Fujii H, Nakai K, et al. Composition and plaque patterns of coronary culprit lesions and clinical characteristics of patients with chronic kidney disease. Kidney. Int. 82(3), 344351(2012).

Stenvinkel P. Inflammation as a target for improving health in chronic kidney disease. F1000 Med. Rep. 17(2), 88 (2010).

Dunne C. Adaptation of bacteria to the intestinal niche: probiotics and gut disorder. Inflamm. Bowel Dis. 7(2), 136145 (2001).

Hooper LV, Gordon JI. Commensal host-bacterial relationships in the gut. Science 292(5519), 1115-1118 (2001).

Bourlioux P, Koletzko B, Guarner F, et al. The intestine and its microflora are partners for the protection of the host: report on the Danone Symposium 'The Intelligent Intestine', held in Paris. $\mathrm{Am} \mathrm{J}$. Clin. Nutr. 78(4), 675-683 (2003).

Cani PD, Delzenne NM. The role of the gut microbiota in energy metabolism and metabolic disease. Curr. Pharm. Des. 15(13), 1546-1558 (2009).

Vaziri ND. CKD impairs barrier function and alters microbial flora of the intestine: a major link to inflammation and uremic toxicity. Curr. Opin. Nephrol. Hypertens. 21(6), 587-592 (2012).

Ley RE, Turnbaugh PJ, Klein S, et al. Microbial ecology: human gut microbes associated with obesity. Nature 444(7122), 1022-1023 (2006).

Marteau P. Bacterial flora in inflammatory bowel disease. Dig. Dis. 27(1), 99-103 (2009).

Vaziri ND, Wong J, Pahl M, et al. Chronic kidney disease alters intestinal microbial flora. Kidney International 83(2), 308-315 (2013).

Kang JY. The gastrointestinal tract in uremia. Dig. Dis. Sci. 38(2), 257-268
(1993).

Wu MJ, Chang CS, Cheng $\mathrm{CH}$, et al. Colonic transit time in long-term dialysis patients. Am. J. Kidney Dis. 44(2), 322327 (2004).

Goraya N, Wesson DE. Dietary management of chronic kidney disease: Protein restriction and beyond. Curr. Opin. Nephrol. Hypertens. 21(6), 635-640 (2012).

Kooman JP, van der Sande FM, Leunissen KM. Role of sodium and volume in the pathogenesis of hypertension in dialysis patients. Reflections on pathophysiological mechanisms. Blood Purif. 22(1), 55-59 (2004).

Werner T, Wagner SJ, Martínez I, et al. Depletion of luminal iron alters the gut microbiota and prevents Crohn's diseaselike ileitis. Gut 60(3), 325-333 (2011).

Choi SS, Kim Y, Han KS, et al. Effects of Lactobacillus strains on cancer cell proliferation and oxidative stress in vitro. Lett. Appl. Microbiol. 42(5), 452-458 (2006).

Nguyen TD, Kang JH, Lee MS. Characterization of Lactobacillus plantarum PH04, a potential probiotic bacterium with cholesterol-lowering effects. Int. J. Food Microbiol. 113(3), 358361 (2007).

Lievin-Le Moal V, Servin AL. Antiinfective activities of lactobacillus strains in the human intestinal microbiota: from probiotics to gastrointestinal antiinfectious biotherapeutic agents. Clin. Microbiol. Rev. 27(2), 167-199 (2014).

Lievin-Le Moal V, Amsellem R, Servin AL, Coconnier MH. Lactobacillus acidophilus (strain LB) from the resident adult human gastrointestinal microflora exerts activity against brush border damage promoted by a diarrhoeagenic Escherichia coli in human enterocyte-like cells. Gut. 50(6), 803-811 (2002).

KDIGO 2012 Clinical Practice Guideline for the Evaluation and Management of Chronic Kidney Disease, Kidney international supplements: the official journal of the international Society of nephrology 3(1), S1-163 (2012).
Mamiatis T, Fritsch EF, Sambrook J, Engel J. Molecular cloning: a laboratory manual. New York: Cold Spring Harbor Laboratory. Acta Biotechnol. 5(1), (1989).

Dubernet S, Desmasures N, Gueguen M. A PCR-based method for identification of lactobacilli at the genus level. FEMS Microbiol. Lett. 214(2), 271-275 (2002).

Sekirov I, Russell SL, Antunes LC et al. Gut microbiota in health and disease. Physiol. Rev. 90(3), 859-904 (2010).

Guldris SC, Parra EG and Amenos AC. Gut microbiota in chronic kidney disease. Nefrologia 37(1), 9-19 (2017).

Li J, Fu R, Yang Y, et al. A metagenomic approach to dissect the genetic composition of enterotypes in Han Chinese and two Muslim groups. Syst. Appl. Microbiol. S0723-2020(17), 30137-30146 (2017).

Hida M, Aiba Y, Sawamura S, et al. Inhibition of the accumulation of uremic toxins in the blood and their precursors in the feces after oral administration of Lebenin, a lactic acid bacteria preparation, to uremic patients undergoing hemodialysis. Nephron. 74(2), 349-355 (1996).

Wang IK, Lai HC, Yu CJ, et al. Real-time PCR analysis of the intestinal microbiotas in peritoneal dialysis patients. Appl. Environ. Microbiol. 78(4), $1107-$ 1112 (2012).

Wong J, Piceno YM, Desantis TZ, et al. Expansion of urease and uricase containing, indole- and p-cresol-forming and contraction of short chain fatty acidproducing intestinal bacteria in ESRD. Am. J. Nephrol. 39(3), 230-237 (2014).

Mafra D, Lobo JC, Barros AF, et al. Role of altered intestinal microbiota in systemic inflammation and cardiovascular disease in chronic kidney disease. Future Microbiol. 9(3), 399-441 (2014).

Yoshifuji A, Wakino S, Irie J, et al. Gut Lactobacillus protects against the progression of renal damage by modulating the gut environment in rats. Nephrol. Dial. Transplant 31(3), 401-412 (2016).

Ramezani A, Massy ZA, Meijers B, 
et al. Role of the gut microbiome in uremia: A potential therapeutic target. Am. J. Kidney Dis. 67(3), 483-498 (2016).

Wing MR, Patel SS, Ramezani A, Raj DS. Gut microbiome in chronic kidney disease. Exp. Physiol. 101(4), 471-477 (2016).

Liabeuf S, Barreto DV, Barreto FC, et al. Free p-cresyl sulphate is a predictor of mortality in patients at different stages of chronic kidney disease. Nephrol. Dial. Transplant. 25(4), 1183-1191 (2015).

Lin CJ, Liu HL, Pan CF, et al. Indoxyl sulfate predicts cardiovascular disease and renal function deterioration in advanced chronic kidney disease. Arch. Med. Res. 43(6), 451-456 (2012).

Celik T, Lyisoy A, Yuksel UC, Jata B. The small intestine: a critical linkage in pathophysiology of cardiac cachexia. Int. J. Cardiol. 143(2), 200-216 (2010).

Sandek A, Valentova M, von Haehling S, DoehnerW, Anker SD. The small intestine: A critical linkage of pathophsiology of cardiac cachexia. Int. J. Cardiol. 146(2), 277-278 (2011).
Koppe L, Mafra D, Fouque D. Probiotics and chronic kidney disease. Kidney Int. 88(5), 958-966 (2015).

Ranganathan N, Patel B, Ranganathan $\mathrm{P}$, et al. Probiotic amelioration of azotemia in 5/6th nephrectomized Sprague-Dawley rats. Scientific World Journal 24(5), 652-660 (2005).

Hyun HS, Paik KH, Cho HY. p-Cresyl sulfate and indoxyl sulfate in pediatric patients on chronic dialysis. Korean. J. Pediatr. 56(4), 159-164 (2013).

Wang IK, Wu YY, Yang YF, et al. The effect of probiotics on serum levels of cytokine and endotoxin in peritoneal dialysis patients: a randomised, doubleblind, placebo-controlled trial. Benef. Microbes. 6(4), 423-430 (2015).

Meijers BK, De PreterV, Verbeke Ket al. p-Cresyl sulfate serum concentrations in haemodialysis patients are reduced by the prebiotic oligofructose-enriched inulin. Nephrol. Dial. Transplant. 25(1), 219-224 (2010).

Vaziri ND, Liu SM, Lau WL, et al. High amylose resistant starch diet ameliorates oxidative stress, inflammation, and progression of chronic kidney disease. PLoS One. 9(12), e114881 (2014).

Moradi H, Vaziri ND, Kashyap ML, Said HM, Kalantar-Zadeh K. Role of HDL dysfunction in end-stage renal disease: a double-edged sword. J. Ren. Nutr. 23(3), 203-206 (2013).

Imhann F, Vich Vila A, Bonder MJ, et al. The influence of proton pump inhibitors and other commonly used medication on the gut microbiota. Gut Microbes. 8(4), 351-358 (2017).

Kortman GA, Raffatellu M, Swinkels DW, Tjalsma H. Nutritional iron turned inside out: intestinal stress from a gut microbial perspective. FEMS Microbiol. Rev. 38(6), 1202-1234 (2014).

Weiss G. Dietary iron supplementation: a proinflammatory attack on the intestine? Gut. 64(5), 696697 (2015) 